\title{
Spatial Clustering with Obstacles Constraints Using Particle Swarm Optimization
}

\author{
Xueping Zhang ${ }^{1,2,3}$ Jiayao Wang ${ }^{2}$ Hongmei Zhang ${ }^{1}$ Jianzhong Guo ${ }^{2} \quad$ Xiaoqing $\mathrm{Li}^{1}$ \\ ${ }^{1}$ Information Science \& Engineering \\ Henan University of Technology \\ No.140 Songshan South Road, \\ Zhengzhou, Henan, China, 450052. \\ +86-371-67756523 \\ zhang_xpcn@yahoo.com.cn \\ ${ }^{2}$ Surveying \& Mapping \\ Information Engineering University \\ No.66 Longhai Middle Road, \\ Zhengzhou, Henan, China, 450052. \\ $+86-371-63535308$ \\ wangjy@ce.com \\ ${ }^{3}$ Geomatics \& Laboratory \\ Liaoning Technical University \\ Fuxin,Liaoning, China, 450052. \\ $+86-371-67787336$ \\ zhangxpcn@yahoo.com.cn
}

\begin{abstract}
Spatial clustering is an important research topic in Spatial Data Mining (SDM). Although many methods have been proposed in the literature, very few have taken into account constraints that may be present in the data or constraints on the clustering. These constraints have significant influence on the results of the clustering process of large spatial data. In this paper, we propose a particle swarm optimization (PSO) method for solving Spatial Clustering with Obstacles Constraints (SCOC). We first use the PSO algorithm based MAKLINK graph to obtain the best obstructed path and then propose a novel PSO and K-Medoids method for SCOC, which is called PKSCOC, to cluster spatial data with obstacles constraints. The PKSCOC algorithm can not only give attention to higher local constringency speed and stronger global optimum search, but also get down to the obstacles constraints and practicalities of spatial clustering. The experimental results show that the PKSCOC algorithm is better than Improved K-Medoids SCOC (IKSCOC) in terms of quantization error and has higher convergence speed than Genetic K-Medoids SCOC (GKSCOC).
\end{abstract}

\section{Categories and Subject Descriptors}

H.2.8 [Data mining] [Spatial databases and GIS]

H.3.3 [Clustering], I.5.3 [Algorithms]

\section{General Terms}

Algorithms, Experimentation, Performance.

\section{Keywords}

Spatial Clustering, Obstacles Constraints, MAKLINK Graph, Obstructed Distance, Particle Swarm Optimization

\section{INTRODUCTION}

Permission to make digital or hard copies of all or part of this work for personal or classroom use is granted without fee provided that copies are not made or distributed for profit or commercial advantage and that copies bear this notice and the full citation on the first page. To copy otherwise, or republish, to post on servers or to redistribute to lists, requires prior specific permission and/or a fee.

INFOSCALE 2007, June 6-8, Suzhou, China Copyright (C) 2007 ICST 978-1-59593-757-5 DOI 10.4108/infoscale.2007.206
Spatial clustering is an important research topic in Spatial Data Mining (SDM). Spatial clustering is not only an important effective method but also a prelude of other task for SDM. As reported in surveys on data clustering, clustering methods can be classified into Partitioning approaches, Hierarchical methods, Density-based algorithms, Probabilistic techniques, Graph theoretic, Grid-based algorithms, Model-based approaches, Genetic Algorithms, Fuzzy methods, Rough Set methods etc. Some algorithms have also integrated two or three kinds of clustering methods. As pointed out earlier, these techniques have focused on the performance in terms of effectiveness and efficiency for large databases. However, few of them have taken into account constraints that may be present in the data or constraints on the clustering. These constraints have significant influence on the results of the clustering process of large spatial data.

Spatial clustering with constraints has two kinds of forms [1]. One kind is Spatial Clustering with Obstacles Constraints (SCOC), such as bridge, river, and highway etc. whose impact on the result should be considered in the clustering process. As an example, Figure 1 shows clustering spatial data with physical obstacle constraints. Ignoring the constraints leads to incorrect interpretation of the correlation among data points. The other kind is Spatial Clustering with Handling Operational Constraints [2], it consider some operation limiting conditions in the clustering process. SCOC is mainly discussed in this paper.

To the best of our knowledge, only three clustering algorithms for SCOC have been proposed very recently, that is COD-CLARANS [3], AUTOCLUST+ [4], and DBCluC [5]-[8], and many questions exist in them. COD-CLARANS computes obstructed distance using visibility graph costly and is unfit for large spatial data. In addition, it only gives attention to local constringency. AUTOCLUST+ builds a Delaunay structure for solving SCOC costly and is also unfit for large spatial data. DBCluC cannot run in large high dimensional data sets etc. We developed Genetic KMedoids SCOC (GKSCOC) based on Genetic algorithms (GAs) and Improved K-Medoids SCOC (IKSCOC) in [9]. The effectiveness and efficiency of GKSCOC is better than IKSCOC. But the drawback of GKSCOC is a comparatively slower speed in clustering.

Particle Swarm Optimization (PSO) is a population-based optimization method first proposed by Kennedy and Eberhart in $1995[10,11]$. Compared to GAs, the advantages of PSO are that 
it is easier to implement and there are fewer parameters to be adjusted, and it has higher constringency speed.

Computing obstructed distance is the key to SCOC. We first use the PSO algorithm based MAKLINK graph to obtain the optimal obstructed path. In order to overcome the disadvantage of partitioning approach which only gives attention to local constringency, and keep the advantage of PSO which has stronger global optimum search and higher constringency speed at the same time, we propose a novel PSO and K-Medoids method for SCOC, which is called PKSCOC, to cluster spatial data with obstacles constraints. The experimental results demonstrate the effectiveness and efficiency of the proposed method, which is better than IKSCOC in terms of quantization error and has higher convergence speed than GKSCOC.

The remainder of the paper is organized as follows. Section 2 introduces PSO. Using PSO to get the optimal obstructed path is discussed in Section 3. Section 4 presents PKSCOC. The performances of PKSCOC implementation on synthetic and real datasets are showed in Section 5, and Section 6 concludes the paper.

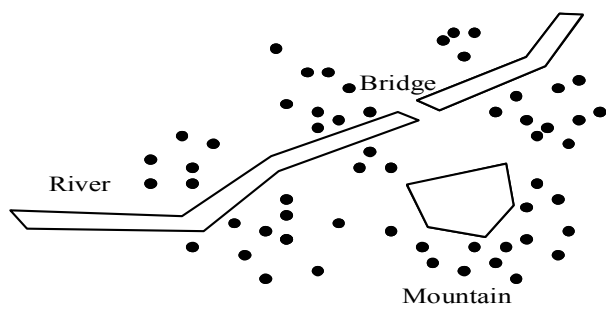

(a) Data Objects and Constraints

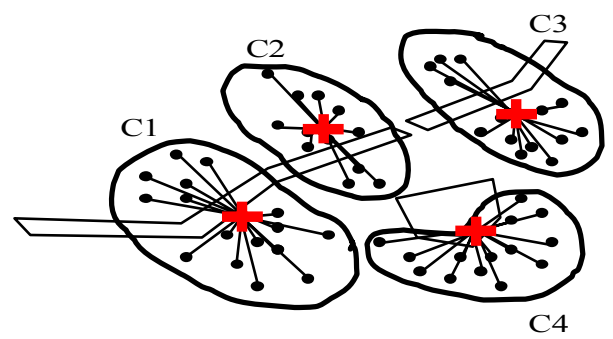

(b) Clusters Ignoring Constraints

Figure 1. Clustering Data Objects with Obstacles Constraints

\section{Particle Swarm Optimization}

Particle Swarm Optimization (PSO) is a population-based optimization method. In order to find an optimal or near-optimal solution to the problem, PSO updates the current generation of particles (each particle is a candidate solution to the problem) using the information about the best solution obtained by each particle and the entire population. The mathematic description of PSO is as the following. Suppose the dimension of the searching space is $\mathrm{D}$, the number of the particles is $\mathrm{n}$. Vector $X_{i}=\left(x_{i 1}, x_{i 2}, \ldots, x_{i D}\right)$ represents the position of the $i^{\text {th }}$ particle and $p$ Best $_{i}=\left(p_{i 1}, p_{i 2}, \ldots, p_{i D}\right)$ is its best position searched by now, and the whole particle swarm's best position is represented as $g$ Best $=\left(g_{1}, g_{2}, \ldots, g_{D}\right)$.Vector $V_{i}=\left(v_{i 1}, v_{i 2}, \ldots, v_{i D}\right)$ is the position change rate of the $i^{\text {th }}$ particle. Each particle updates its position according to the following formulas:

$$
\begin{array}{r}
v_{i d}(t+1)=w^{*} v_{i d}(t)+c_{1} * \operatorname{rand}() *\left[p_{i d}(t)-x_{i d}(t)\right] \\
{ }_{c_{2}} * \operatorname{rand}() *\left[g_{d}(t)-x_{i d}(t)\right] \\
x_{i d}(t+1)=x_{i d}(t)+v_{i d}(t+1), 1 \leq i \leq n, 1 \leq d \leq D
\end{array}
$$

where $w$ is the inertia weight, $c_{1}$ and $c_{2}$ are positive constant parameters, and $\operatorname{Rand}()$ is a random function with the range [0, 1]. Equation (1) is used to calculate the particle's new velocity, then the particle flies toward a new position according to Equation (2). The various range of the $d^{\text {th }}$ position is $\left[X M I N X_{d}, X M A X X_{d}\right]$ and the various range $\left[-V M A X X_{d}, V M A X X_{d}\right]$. If the value calculated by equations (1) and (2) exceeds the range, set it as the boundary value. The performance of each particle is measured according to a predefined fitness function, which is usually proportional to the cost function associated with the problem. This process is repeated until user-defined stopping criteria are satisfied.

PSO is effective in nonlinear optimization problems and it is easy to implement. In addition, only few input parameters need to be adjusted in PSO. Because the update process in PSO is based on simple equations, PSO can be efficiently used on large data sets. A disadvantage of the global PSO is that it tends to be trapped in a local optimum under some initialization conditions [12].

\section{Using PSO to Get the Optimal Obstructed Path}

\subsection{Obstructed distance}

The direct Euclidean distance is always adopted in typical clustering methods to estimate the clustering quality. However, the obstructed distance must be introduced to handle obstacle constraints, and it is can be defined as follows.

Given point $p$ and point $q$, the obstructed distance $d_{o}(p, q)$ is defined as the length of the shortest Euclidean path between two points $p$ and $q$ without cutting through any obstacles.

Path planning with obstacles constraints is the key to computing obstructed distance. In this paper, we adopt a simple model of obstacles called MAKLINK graph [13], which can reduce the complicacy of the model and get the optimized path, for path planning with obstacles constraints.

\subsection{Obstacles Modeling}

The structuring of MAKLINK graph is as the following steps:

1. Find all the lines that connect one of the corners that belong to a polygonal obstacle, with all the other obstacles' corners including the corners of the current obstacle;

2. Delete the redundant free links to make every free space, of which the edges are free links, obstacle edges and boundary walls, be a convex polygon and its area be largest;

3 . Find the midpoint of the remained free links and take them as the path nodes, labeling orderly as $1,2, \cdots, n$. The connections 
among the midpoints that belong to the same convex area compose a network.

Further explanations and detail on how to construction MAKLINK graph can be found in [13]. An example is shown in Figure 2.
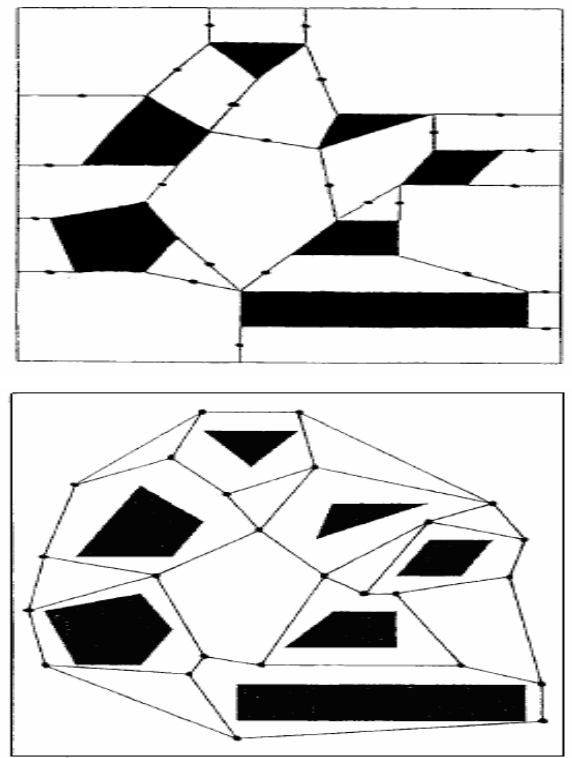

Figure 2. MAKLINK Graph Based on Free Convex Polygon

\subsection{Using Dijkstra Algorithm to Obtain the} Shortest Path

Path planning with obstacles constraints can be divided into two stages [14], that is, first finds out the shortest path from the start point to the goal point in the MAKLINK graph using Dijkstra algorithm, then adopts PSO algorithm to optimize the shortest path and get the best global path. This section introduces using Dijkstra algorithm to obtain the shortest path firstly.

Take the label sequence of the path nodes as a path code. If the start or goal point is not in the MAKLINK graph, connect this point with the path nodes that belong to the same free convex space and take it as a new path node. The lengths of the arcs in the graph are their weights and the definition of the adjust matrix is as the following:

weight $(i, j)=\left\{\begin{array}{cc}w_{i j} & v_{i} \neq v_{j}, \text { and }<v_{i}, v_{i}>\in E(G) \\ 0 & v_{i}=v_{j} \\ \infty & \text { others }\end{array}\right.$

Where $v_{i}$ and $v_{j}$ are any two of the nodes in the graph $E(G)$, $\left.<v_{i}, v_{i}\right\rangle$ represents an arc in the graph and $w_{i j}$ is its weight. The simulation result is in Figure 3 and the black solid line represents the shortest path we got.

\subsection{Using PSO Algorithm to Get the Optimal Obstructed Path}

Suppose the shortest path of the MAKLINK graph that we get by Dijkstra algorithm is $P_{0}, P_{1}, P_{2}, \ldots, P_{D}, P_{D+1}$, where $P_{0}=$ start

is the start point and $P_{D+1}=$ goal is the goal point. $P_{i}(i=1,2, \ldots, D)$ is the midpoint of the free link. The optimization task is to adjust the position of $P_{i}$ to shorten the length of path and get the optimized (or acceptable) path in the planning space. The adjust process of $P_{i}$ is shown in Figure 4 [14]. The position of $P_{i}$ can be decided by the following parametric equation:

$P_{i}=P_{i 1}+\left(P_{i 2}-P_{i 1}\right) \times t_{i}, t_{i} \in[0,1], i=1,2, \ldots D$

Each particle $X_{i}$ is constructed as: $X_{i}=\left(t_{1} t_{2} \ldots t_{D}\right)$.Accordingly, the $i^{\text {th }}$ particle's fitness value is defined as:

$f\left(X_{i}\right)=\sum_{k=1}^{D+1} P_{k-1} P_{k}, i=1,2, \ldots, n$

where $P_{k-1} P_{k}$ is the direct Euclidean distance between the two points and $P_{k}$ can be calculated according to equation (5). Thus the smaller the fitness value is, the better the solution is.

Here, the PSO is adopted as follows.

1. Initialize particles at random, and set $p B e s t_{i}=X_{i}$;

2. Calculate each particle's fitness value according to equation (5) and label the particle with the minimum fitness value as $g$ Best ;

3 . For $t_{1}=1$ to $t_{1} \max$ do \{

4. For each particle $X_{i}$ do \{

5. Update $v_{i d}$ and $x_{i d}$ according to equations (1) and (2);

6. Calculate the fitness according to equation (5);\}

9. Update gBest and pBest ${ }_{i}$;

10. If $\|\mathrm{v}\| \leq \varepsilon$, terminate ;

where $t_{1} \max$ is the maximum number of iterations, $\varepsilon$ is the minimum velocity. The simulation result is in Figure 5 and the red solid line represents the optimal obstructed path obtained by PSO.

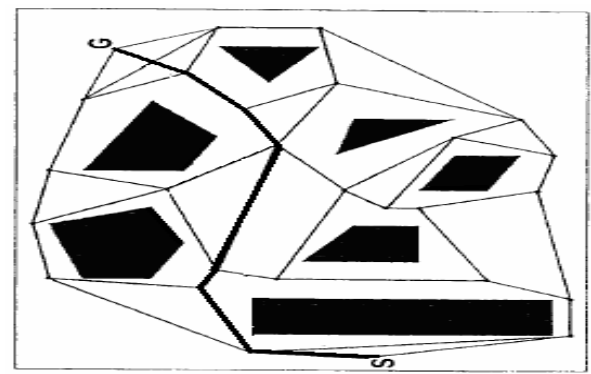

Figure 3. Shortest Obstructed Path 


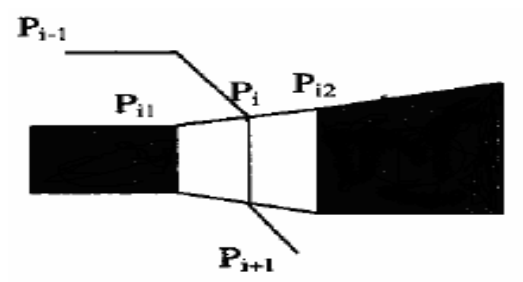

Figure 4. Path Coding Method

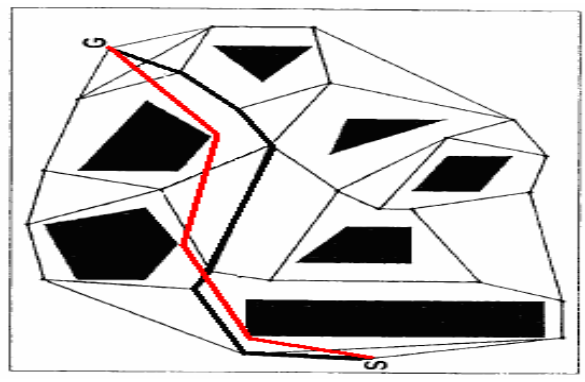

Figure 5. Optimal Obstructed Path

\section{PKSCOC Based on PSO and K-Medoids}

This section first introduces IKSCOC in section 4.1, and then presents the PKSCOC algorithm in section 4.2.

\subsection{IKSCOC Based on K-Medoids}

Partitioning-base algorithm divides $n$ objects into $k(k<n)$ parts, and each part represents one cluster. There are three classic types: K-Means, K-Medoids and CLARANS. K-Means takes the average value of a cluster as the cluster centre. While adopting this algorithm, a cluster center possibly just falls on the obstacle (Figure 6), and it cannot be implemented in reality.
K-Means

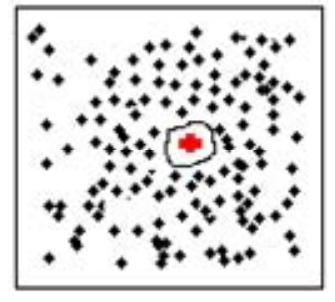

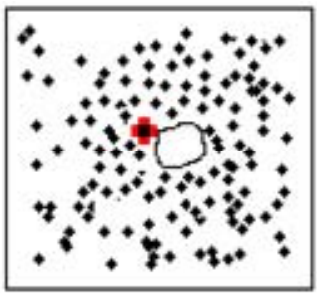

K-Medoids
Figure 6. K-Means vs. K-Medoids

On the other hand, K-Medoids takes the most central object of a cluster as the cluster centre, and the cluster center cannot fall on the obstacle. In view of this, K-Medoids algorithm is adopted for SCOC to avoid cluster center falling on the obstacle.

The clustering quality is estimated by an object function. Squareerror function is adopted here, and its definition can be defined as:

$$
E=\sum_{j=1}^{N_{c}} \sum_{p \in C_{j}}\left(d\left(p, m_{j}\right)\right)^{2}
$$

where $N_{c}$ is the number of cluster $C_{j}, m_{j}$ is the cluster centre of cluster $C_{j}, d(p, q)$ is the direct Euclidean distance between the two points $p$ and $q$.

To handle obstacle constraints, accordingly, criterion function for estimating the quality of spatial clustering with obstacles constraints can be revised as:

$$
E_{o}=\sum_{j=1}^{N_{c}} \sum_{p \in C_{j}}\left(d_{o}\left(p, m_{j}\right)\right)^{2}
$$

where $d_{o}(p, q)$ is the obstructed distance between point $p$ and point $q$.

The method of IKSCOC is proposed as follows [9].

1. Select $N_{c}$ objects to be cluster centers at random;

2. Distribute remain objects to the nearest cluster center;

3. Calculate $E_{o}$ according to equation (7);

4. Do \{let current $E=E_{o}$;

5. Select a not centering point to replace the cluster center $m_{j}$ randomly;

6. Distribute objects to the nearest center;

7. Calculate $E$ according to equation (6);

8. If $E>$ current $E$, go to 5;

9. Calculate $E_{o}$;

10. If $E_{o}<$ current $E$, form new cluster centers;

11.) While ( $E_{o}$ changed).

While IKSCOC still inherits two shortcomings because it is based on standard partitioning algorithm. One shortcoming is that selecting initial value randomly may cause different results of the spatial clustering and even have no solution. The other is that it only gives attention to local constringency and is sensitive to an outlier.

\subsection{PKSCOC Based on PSO and K-Medoids}

Particle Swarm Optimization (PSO) has been applied to data clustering [15-18]. In the context of clustering, a single particle represents the $N_{c}$ cluster centroid. That is, each particle $X_{i}$ is constructed as follows:

$X_{i}=\left(m_{i 1}, \ldots, m_{i j}, \ldots, m_{i N d}\right)$

where $N_{d}$ refers to the input dimension, $m_{i j}$ refers to the $j^{\text {th }}$ cluster centroid of the $i^{\text {th }}$ particle in cluster $C_{i j}$. Here, the objective function is defined as follows:

$$
f\left(x_{i}\right)=\frac{1}{J_{i}}
$$




$$
J_{i}=\sum_{j=1}^{N_{c}} \sum_{p \in C_{i j}} d_{o}\left(p, m_{j}\right)
$$

The lower $J_{i}$ is, the higher the fitness value is.

Using the standard gBest PSO, Spatial Clustering with Obstacles Constraints based on PSO and K-Medoids (PKSCOC), which absorbs the though of the K-means PSO hybrid as presented in [16], is developed as follows.

1. Execute the IKSCOC algorithm to initialize one particle to contain $N_{C}$ selected cluster centroids;

2. Initialize the other particles of the swarm to contain $N_{c}$ selected cluster centroids at random;

3 . For $t=1$ to $t_{\max }$ do \{

4. For each particle $i$ do \{

5. For each object $p$ do \{

6. Calculate $d_{o}\left(p, m_{i j}\right)$;

7. Assign object $p$ to cluster $C_{i j}$ such that

$$
d_{o}\left(p, m_{i j}\right)=\min \forall c=1, \ldots, N_{d}\left\{d_{o}\left(p, m_{i c}\right)\right\} \text {; }
$$

8. Calculate the fitness according to equation (9) ; \} \}

9. Update $g$ Best and $p$ Best $_{i}$;

10. Update the cluster centroids according to equation (1) and equation (2);

11 If $\|\mathrm{v}\| \leq \varepsilon$, terminate;

12. Optimize new individuals using the IKSCOC algorithm ;\}

where $t_{\text {max }}$ is the maximum number of iteration, $\varepsilon$ is the minimum velocity. STEP 1 is to overcome the disadvantage of the global PSO which tends to be trapped in a local optimum under some initialization conditions. STEP 12 is to improve the local constringency speed of the global PSO.

The population-based search of the PKSCOC algorithm reduces the effect that initial conditions have, as opposed to the IKSCOC algorithm; the search starts from multiple positions in parallel. Section 5 shows the effectiveness and efficiency of the PKSCOC algorithm

\section{Results and Discussion}

This section presents experimental results on synthetic and real datasets. We have made experiments separately by K-Medoids, IKSCOC, GKSCOC, and PKSCOC. $n=50, w=0.72, c_{1}=c_{2}=2, V_{\max }=0.4, t_{\max }=100, \omega=0.001$.

Figure 7 shows the results on synthetic Dataset1. Figure 7 (a) shows the original data with simple obstacles. Figure 7 (b) shows the results of 4 clusters found by K-Medoids without considering obstacles constraints. Figure 7(c), Figure 7 (d), and Figure 7 (e) shows 4 clusters found by IKSCOC, GKSCOC, and PKSCOC respectively. Obviously, the results of the clustering illustrated in Figure 7(c), Figure 7 (d), and Figure 7 (e) all have better practicalities than that in Figure 7 (b). And the one in Figure 7 (e) is superior to the one in Figure 7 (c) but is less inferior to the one in Figure 7 (d).

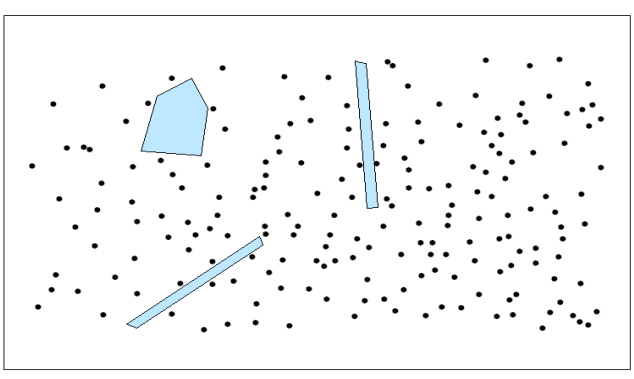

(a)

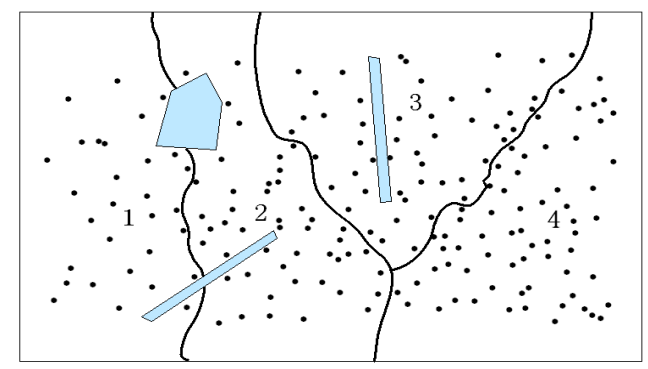

(b)

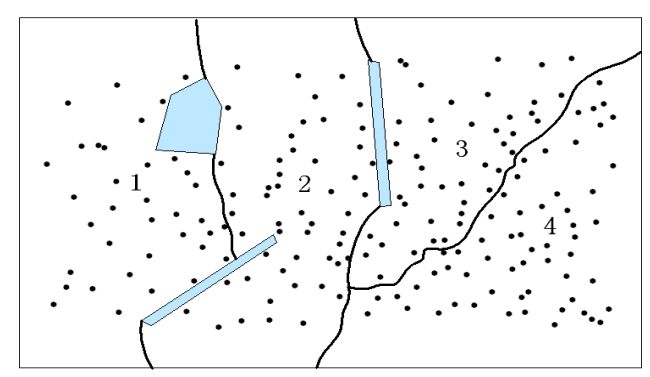

(c)

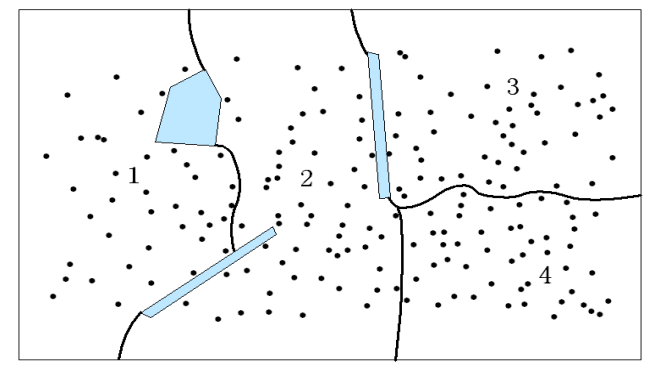

(d)

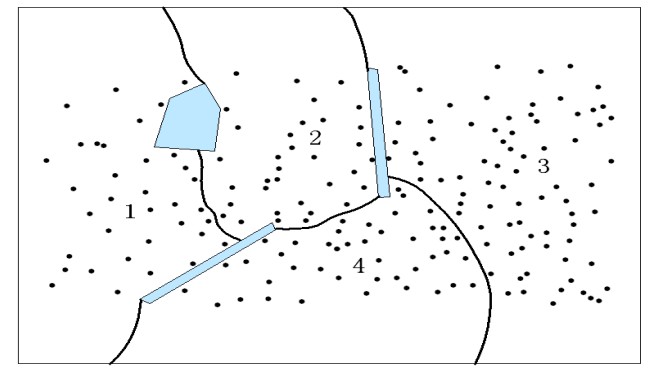

(e)

Figure 7. Clustering Dataset1 
Figure 8 shows the results on synthetic Dataset2. Figure 8(a) shows the original data with various obstacles. Figure 8(b) shows 4 clusters found by K-Medoids. Figure 8(c) shows 4 clusters found by PKSCOC. Obviously, the result of the clustering illustrated in Figure 8(c) has better practicalities than the one in Figure 8(b). So, it can be drawn that PKSCOC is effective for various obstacles.

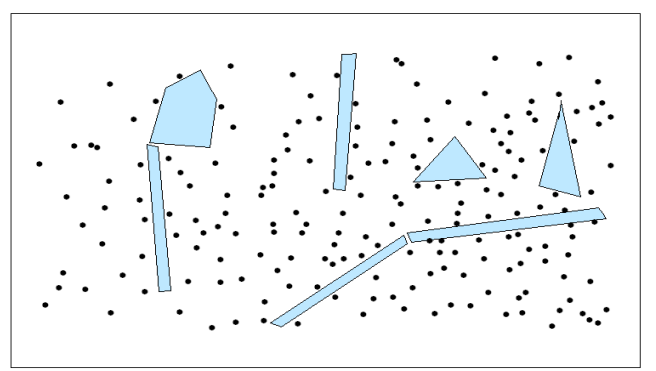

(a)

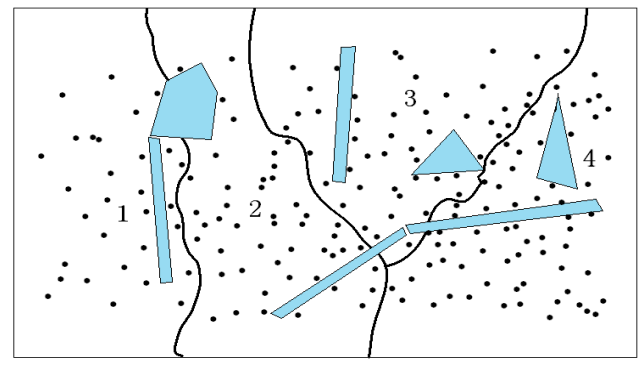

(b)

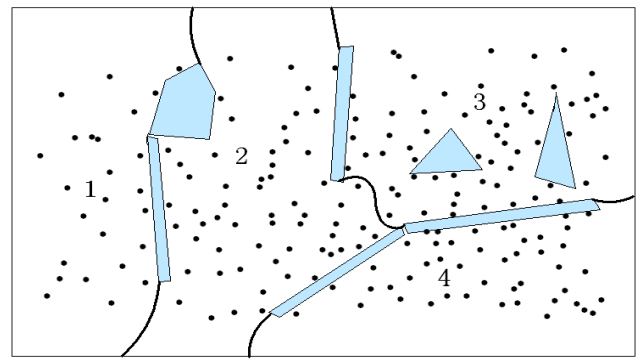

(c)

Figure 8. Clustering Dataset2

Figure 9 shows the results on real Dataset3. Figure 9(a) shows the original data with the Yellow River obstacles. Figure 9(b), Figure 9(c), Figure 9(d), and Figure 9(e) shows 4 clusters found by KMedoids, IKSCOC, GKSCOC, and PKSCOC respectively. Obviously, the results of the clustering illustrated in Figure 9(c), Figure 9(d), and Figure 9(e) all have better practicalities than that in Figure 9(b), and the one in Figure 9(e) is superior to the one in Figure 9(c).

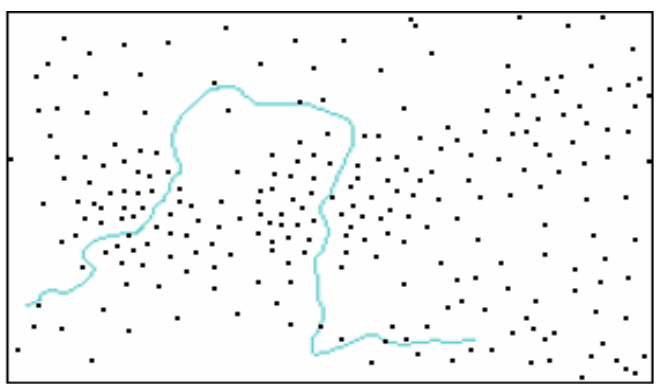

(a)

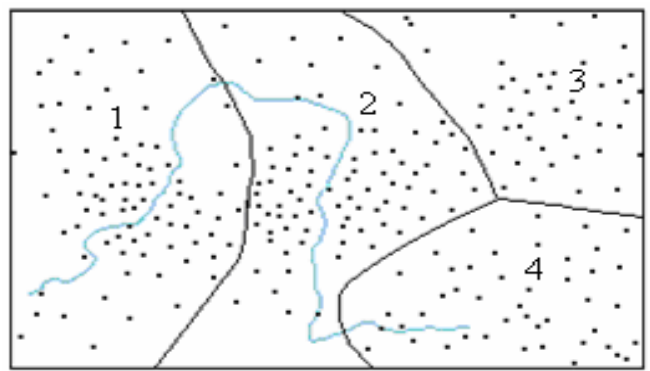

(b)

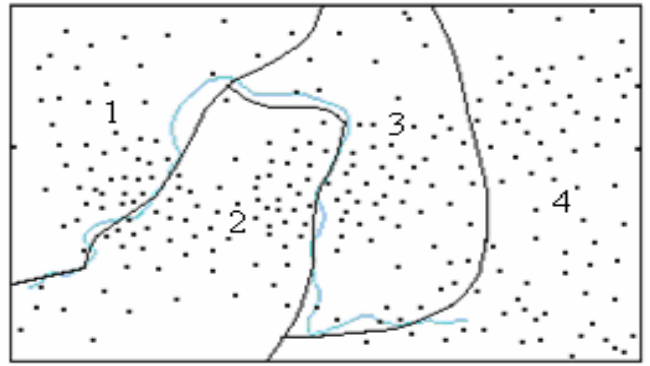

(c)

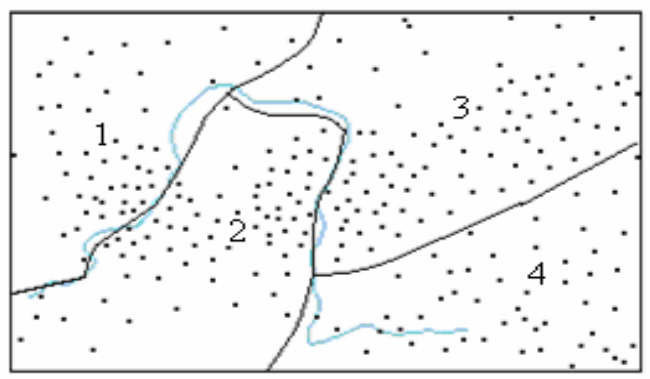

(d)

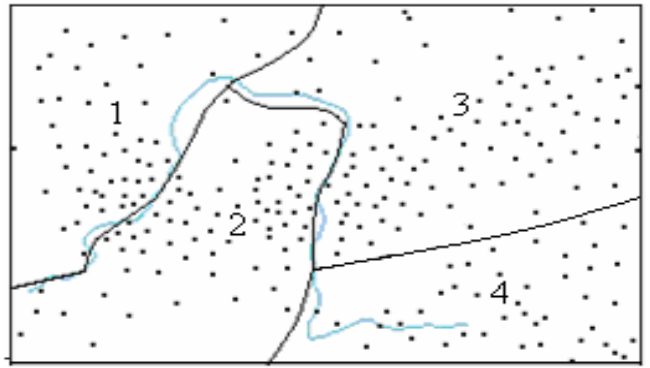

(e)

Figure 9. Clustering Dataset3 
Figure 10 is the constringency speed in one experiment on Dataset1. It is showed that PKSCOC constringes in about 12 generations while GKSCOC constringes in nearly 25 generations. So, it can be drawn that PKSCOC is effective and has higher constringency speed than GKSCOC.

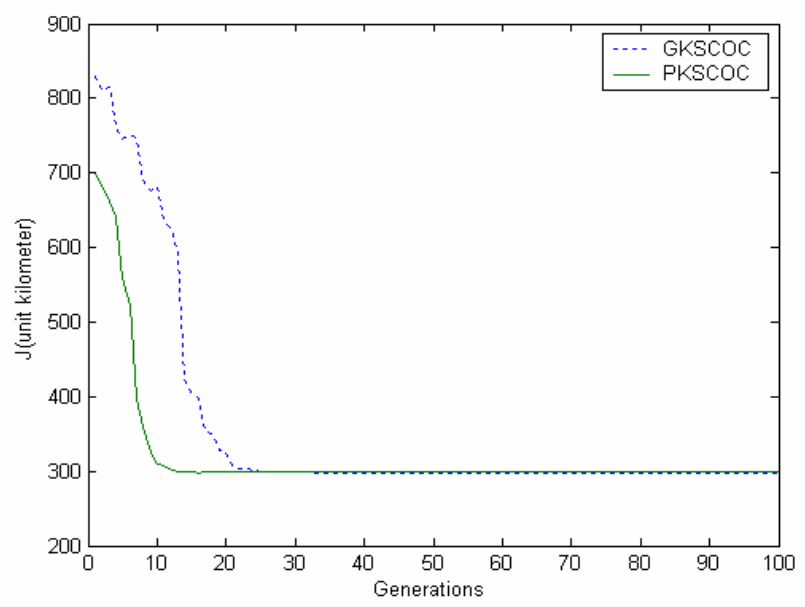

Figure 10. PKSCOC vs. GKSCOC

Figure 11 is the value of $J$ showed in every experiment on Dataset 1 by IKSCOC and PKSCOC respectively. It is showed that IKSCOC is sensitive to initial value and it constringes in different extremely local optimum points by starting at different initial value while PKSCOC constringes nearly in the same optimum points at each time. Therefore, we can draw the conclusion that PKSCOC has stronger global constringent ability than IKSCOC and has higher convergence speed than GKSCOC.

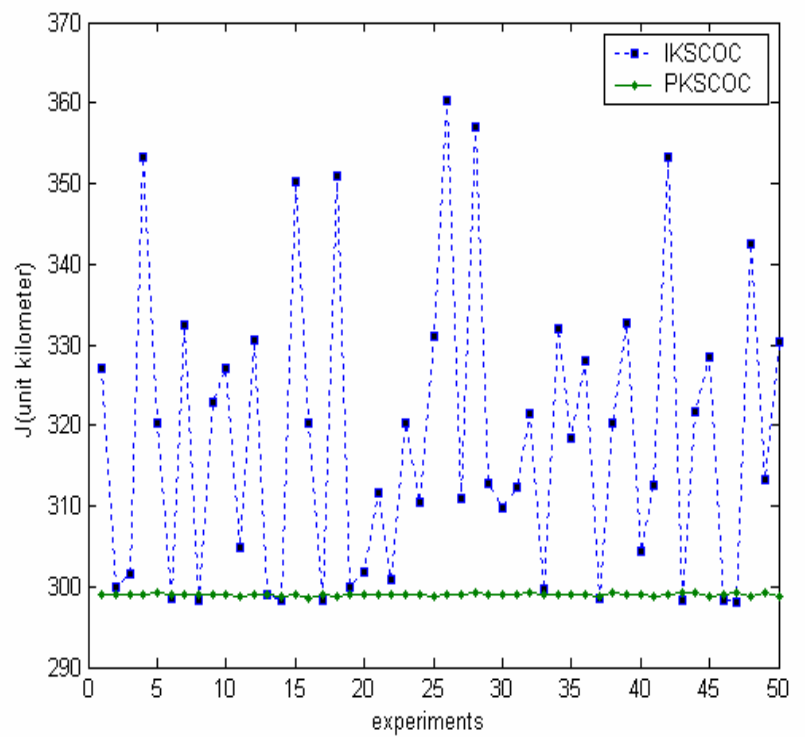

Figure 11. PKSCOC vs. IKSCOC

\section{Conclusions}

Spatial clustering is not only an important effective method, but also a prelude of other task for Spatial Data Mining (SDM). Classic clustering algorithms have ignored the fact that many constraints exit in the real world and could affect the effectiveness of clustering result. This paper proposes a PSO method for solving SCOC. In the process of doing so, we use the PSO algorithm based MAKLINK graph to obtain the best obstructed path and develop a novel PKSCOC based on PSO and K-Medoids to cluster spatial data with obstacles constraints. The PKSCOC algorithm can not only give attention to higher local constringency speed and stronger global optimum search, but also get down to the obstacles constraints and practicalities of spatial clustering. The experimental results demonstrate the effectiveness and efficiency of the proposed method, which performs better than IKSCOC in terms of quantization error and has higher convergence speed than GKSCOC. And its achievements will have more practical value and extensive application prospect.

\section{Acknowledgments}

This work is partially supported by the Natural Sciences Fund Council of China (Number: 40471115) , the Natural Sciences Fund of Henan (Number:0511011000, Number: 0624220081), and the Open Research Fund Program of the Geomatics and Applications Laboratory, Liaoning Technical University (Number: 2004010)

\section{References}

[1] A.K.H.Tung, J.Han, L.V.S.Lakshmanan, and R.T.Ng. Constraint-Based Clustering in Large Databases. In Proceedings of the International Conference on Database Theory (ICDT'01). London, U.K., 2001, 405-419.

[2] A.K.H.Tung, R.T.Ng, L.V.S.Lakshmanan, and J.Han. Geospatial Clustering with User-Specified Constraints. In Proceedings of the International Workshop on Multimedia Data Mining (MDM/KDD'2000), in conjunction with ACM SIGKDD conference. Boston, USA, August 20, 2000, 1-7.

[3] A.K.H.Tung, J.Hou, and J.Han. Spatial Clustering in the Presence of Obstacles. In Proceedings of International Conference on Data Engineering (ICDE'01). Heidelberg, Germany, April, 2001, 359-367.

[4] V.Estivill-Castro and I.J.Lee. AUTOCLUST+: Automatic Clustering of Point-Data Sets in the Presence of Obstacles. In Proceedings of the International Workshop on Temporal, Spatial and Spatial-Temporal Data Mining. Lyon, France, 2000, 133-146.

[5] O.R.Zaïane and C. H.Lee. Clustering Spatial Data When Facing Physical Constraints. In Proceedings of the IEEE International Conference on Data Mining (ICDM'02). Maebashi City, Japan, 2002, 737-740.

[6] X.Wang, H.J.Hamilton. DBRS: A Density-Based Spatial Clustering Method with Random Sampling. In Proceedings of the 7th PAKDD. Seoul, Korea, 2003, 563- 575.

[7] X.Wang,C.Rostoker,H.J.Hamilton. DBRS+:Density-Based Spatial Clustering in the Presence of Obstacles and Facilitators. ftp.cs.uregina.ca/Research/Techreports/200409.pdf, 2004.

[8] X.Wang, H.J.Hamilton. Gen and SynGeoDataGen Data Generators for Obstacle Facilitator Constrained Clustering. ftp.cs.uregina.ca/Research/Techreports/2004-08.pdf, 2004. 
[9] Xueping Zhang, Jiayao Wang, Fang Wu, Zhongshan Fan and Xiaoqing Li. A Novel Spatial Clustering with Obstacles Constraints Based on Genetic Algorithms and K-Medoids. In Proceedings of the Sixth International Conference on Intelligent Systems Design and Applications (ISDA 2006). Jinan, Shangdong,China, October,2006, 605-610.

[10] Russ C. Eberhart and J. Kennedy. A new optimizer using particle swarm theory. In Proceedings of the Sixth International Symposium on Micro Machine and Human Science. Nagoya Japan, 1995, 39-43.

[11] J. Kennedy and R. C. Eberhart. Particle Swarm Optimization. In Proceedings of IEEE International Conference on Neural Networks. Volume IV, Perth Australia ,1995,1942-1948.

[12] Frans van den Bergh. An Analysis of Particle Swarm Optimizers. Ph.D. thesis, University of Pretoria, 2001.

[13] Maki K. HABIB, Hajime ASAMA. Efficient method to generate collision free paths for autonomous mobile robot based on new free space structuring approach. In Proceedings of International Workshop on Intelligent Robots and Systems. Japan, November, 1991, 563-567.

[14] Yuanqing Qin, Debao Sun, Ning Li, Yigang Cen. Path planning for mobile robot using the particle swarm optimization with mutation operator. In Proceedings of the Third International Conference on Machine Learning and Cybernetics. Shanghai,China, August, 2004,2473-2478.

[15] Xiang Xiao Dow, E.R. Eberhart, R. Miled, Z.B. Oppelt, R.J. Gene Clustering Using Self-Organizing Maps and Particle Swarm Optimization. In Proceedings of the International Conference on Parallel and Distributed Processing Symposium (IPDPS), 2003.

[16] Van der Merwe DW,Engelbrecht A P. Data Clustering Using Particle Swarm Optimization. In Proceedings of IEEE Congress on Evolutionary Computation 2003 (CEC 2003).2003, 215-220.

[17] MGH Omran. Particle Swarm Optimization Methods for Pattern Recognition and Image Processing. Ph.D. thesis, University of Pretoria, 2005.

[18] Xiaohui Cui,Potok T.E.,Palathingal P. Document clustering using particle swarm optimization. In Proceedings of IEEE on Swarm Intelligence Symposium (SIS 2005). 2005,185-191.

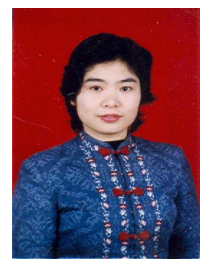

Xueping Zhang received the B.S. and M.S. degrees in Computer Science from ZhengZhou University in 1990 and 2001, respectively. After working as an assistant professor (from 1996) in the School of Computer Science and Engineering at Henan University of Technology, she has been an associate professor at Henan University of Technology since 2002. Her research interest includes Data Mining, Artificial Intelligence and Software Engineering, and their application to GIS. Now, she is a PhD student in GIS in the Institute of Surveying and Mapping, PLA Information Engineering University.

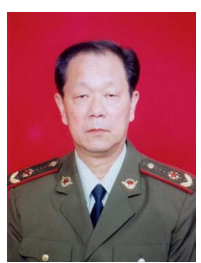

Jiayao Wang is a member of Chinese Academy of Engineering, and he is a professor in the Institute of Surveying and Mapping at PLA Information Engineering University. His research interest includes Theoretical Cartography, and Spatial Information System.

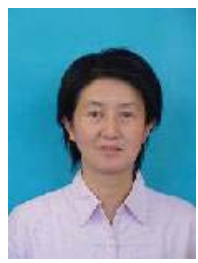

Hongmei Zhang is a professor in the School of Computer Science and Engineering at Henan University of Technology. Her research interest includes Image Processing, Artificial Intelligence, and Software Engineering.

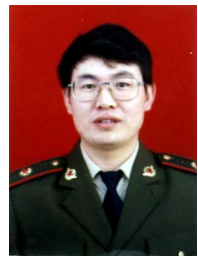

Jianzhong Guo is an associate professor in the Institute of Surveying and Mapping at PLA Information Engineering University. His research interest includes Theoretical Cartography, GIS, and so on.

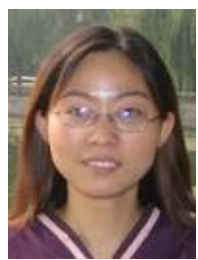

Xiaoqing $\mathbf{L i}$ is a M.S. student in the School of Computer Science and Engineering at Henan University of Technology. Her research interests are Data Mining and so on. 